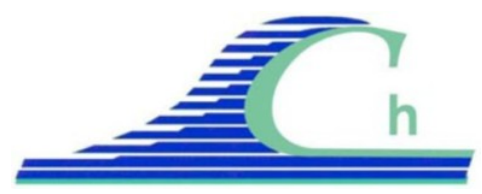

XII ${ }^{\text {èmes }}$ Journées Nationales Génie Côtier - Génie Civil

Cherbourg, 12-14 juin 2012

DOI:10.5150/jngcgc.2012.050-S @ Editions Paralia CFL

disponible en ligne - http://www.paralia.fr - available online

\title{
Dynamique morphosédimentaire des cordons dunaires et cinématique du trait de côte en contexte macrotidal
}

\author{
Serge SUANEZ ${ }^{1}$, Jean-Marie CARIOLET ${ }^{2}$, Bernard FICHAUT $^{1}$
}

1. Université de Bretagne Occidentale, IUEM, UMR 6554 CNRS LETG-Géomer, Technopôle Brest Iroise, Place Nicolas Copernic, 29280 Plouzané, France. serge.suanez@univ-brest.fr ; bernard.fichaut@univ-brest.fr

2. Department of Geography, Bennett Building, University of Leicester, Leicester LE1 7RH, UK.

jc484@leicester.ac.uk

\section{Résumé :}

En contexte macrotidal, la ligne de référence du trait de côte géomorphologique correspond le plus souvent à la limite de végétation dunaire. Cette dernière peut également coïncider avec la crête de dune lorsque le versant externe présente une falaise d'érosion. En période de régénération, la construction de dunes embryonnaires au pied de cordon, surtout lorsque ce dernier culmine à une dizaine de mètres de hauteur, ne permet plus de conserver le même référentiel. Les dynamiques morphosédimentaire et végétale des nouveaux bourrelets dunaires doivent être alors considérées.

Mots-clefs :

Trait de côte - Végétation dunaire - Dune embryonnaire - Régénération dunaire

\section{Introduction}

Depuis le milieu du $19^{\text {ème }}$ siècle, en France comme aux Etats-Unis, la ligne de référence du trait de côte retenue par les services de l'Etat dans l'établissement des cartes topographiques ou bathymétriques correspond à la limite des pleines mers de vive-eau. Ce référentiel a l'avantage d'être toujours homogène quel que soit le type de côte et de marnage, mais suppose des traitements assez lourds, notamment pour filtrer les effets de la marée (ALLAIN et al., 2000 ; LEATHERMAN, 2003). En géomorphologie, le trait de côte repose sur une limite physiographique "fixe" que l'on peut suivre dans le temps et dans l'espace, mais dans ce cas, elle diffère suivant le type de côte et le contexte marégraphique (ROBIN, 2002 ; LE BERRE et al., 2005). Les synthèses réalisées à partir de travaux portant sur l'analyse de la cinématique du trait de côte montrent qu'il existe plusieurs dizaines de référentiels pouvant être classées en trois grandes familles (GRENIER \& DUBOIS, 1990 ; ROBIN, 2002 ; BOAK \& TURNER, 2005) : (i) les indicateurs morphologiques (sommet de falaises, crête ou pied de dune, ados de plage, etc.) ; (ii) les indicateurs biologiques (limite de végétation dunaire ou intertidale); (iii) la position des différents niveaux d'eau (niveau marin instantané, moyen ou de pleine mer de vive-eau, limite maximum du jet de rive, ligne d'exfiltration 
sur l'estran). Sur les côtes d'accumulation sableuse de type macro à mégatidal, la limite du trait de côte correspond le plus souvent au contact entre la végétation dunaire et les sables vifs du haut de plage intertidale (GAILLOT \& CHAVEROT, 2001 ; LE BERRE et al., 2005; SUANEZ \& STÉPHAN, 2006; SUANEZ et al., 2010 ; SUANEZ \& STÉPHAN, 2011). L'objectif de cet article est de faire une mise au point sur la définition du trait de côte des cordons littoraux sableux en milieu macrotidal en tenant compte des processus morphodynamiques des dunes qui agissent sous l'action érosive lors des épisodes morphogènes ou de régénération en période d'accalmie. L'analyse porte sur un secteur littoral situé dans le nord Finistère, et s'étend sur la période allant du printemps 2008 à l'été 2011. Elle a débuté par la tempête Johanna du 10 mars 2008, dont les effets ont été particulièrement morphogènes, et s'est poursuivie par une longue phase de régénération dunaire. Le travail repose sur un suivi du bilan sédimentaire et de la cinématique du trait de côte défini dans cette étude par la limite de végétation dunaire.

\section{Le site d'étude et les méthodes d'analyse}

La zone d'étude correspond à la plage du Vougot située sur la côte nord du Finistère (figure 1). Il s'agit d'un cordon sableux d'âge holocène dont la construction a été facilitée par la présence d'une large plateforme à écueils s'étendant en contrebas du plateau du Léon qui a bloqué le matériel sédimentaire poussé par la mer lors de la transgression flandrienne (GUILCHER \& HALLÉGOUËT, 1991).

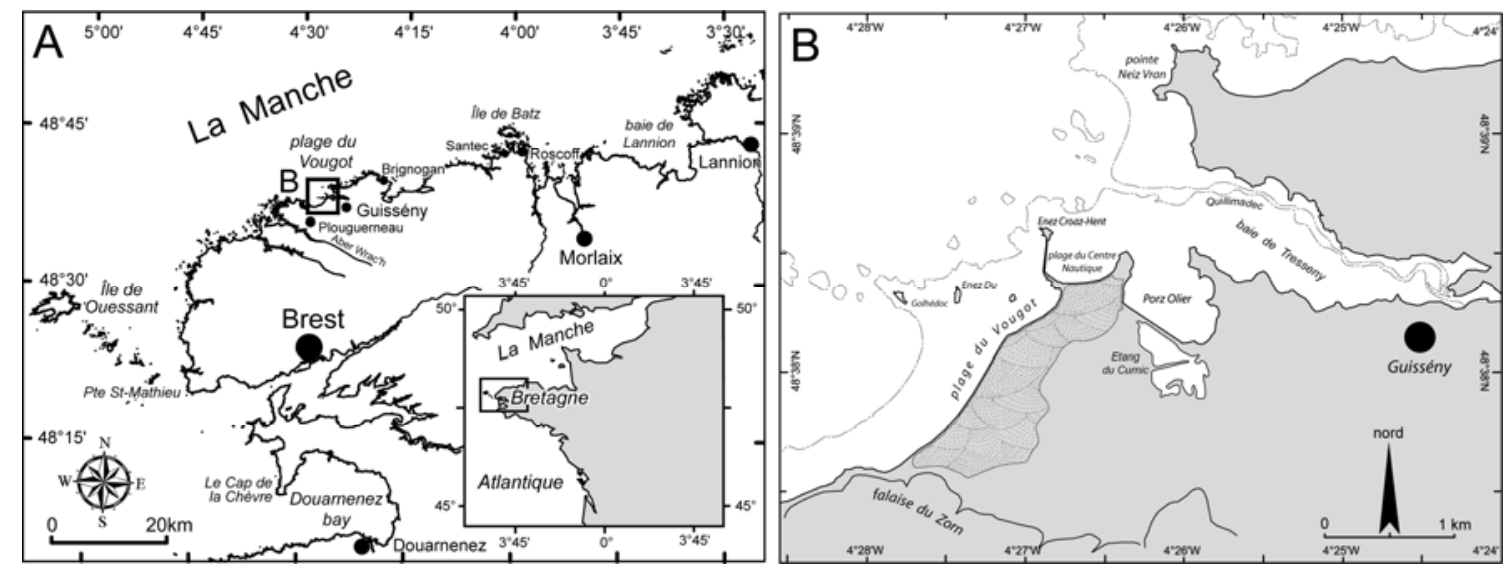

Figure 1. Carte de localisation régionale (A) et locale (B) plage du Vougot.

Ce cordon littoral est formé d'un vaste édifice dunaire qui s'étire sur $2 \mathrm{~km}$ depuis la falaise morte du Zorn, suivant une direction sud-ouest nord-est. Cette dune culmine à près de $13 \mathrm{~m}$ d'altitude NGF et se présente sous la forme d'un complexe dunaire massif d'une largeur comprise entre $250 \mathrm{~m}$ et $400 \mathrm{~m}$. L'évolution morphosédimentaire de cette dune, notamment dans sa partie orientale, montre une tendance érosive depuis les années 1970 (SUANEZ et al., 2010). 


\section{XII ${ }^{\text {èmes }}$ Journées Nationales Génie Côtier - Génie Civil \\ Cherbourg, 12-14 juin 2012}

Cette étude repose sur un ensemble de mesures de terrain réalisées au DGPS qui ont été a été raccordées au système géodésique français. Il s'agit d'analyser la cinématique du trait de côte et de quantifier le bilan sédimentaire à partir de mesures de profil de plage/dune et/ou de levés topo-morphologiques surfaciques (MNT). Dans le cas du suivi de la cinématique du trait de côte, la ligne de référence qui a été utilisée correspond au sommet du front de dune. Le choix de cet indicateur repose sur le fait que depuis 2004, date à laquelle les mesures de terrain ont commencé, cette limite est clairement identifiée par la présence d'un versant externe de la dune très redressé et non végétalisé (figure 2). Cet abrupt subvertical correspondant à une falaise d'érosion dunaire illustre la tendance érosive pluridécennale de cette partie du cordon dunaire (SUANEZ et al., 2010). Il marque également la limite franche de végétation dunaire entre la dune grise et les sables vifs du front de dune/haut d'estran.
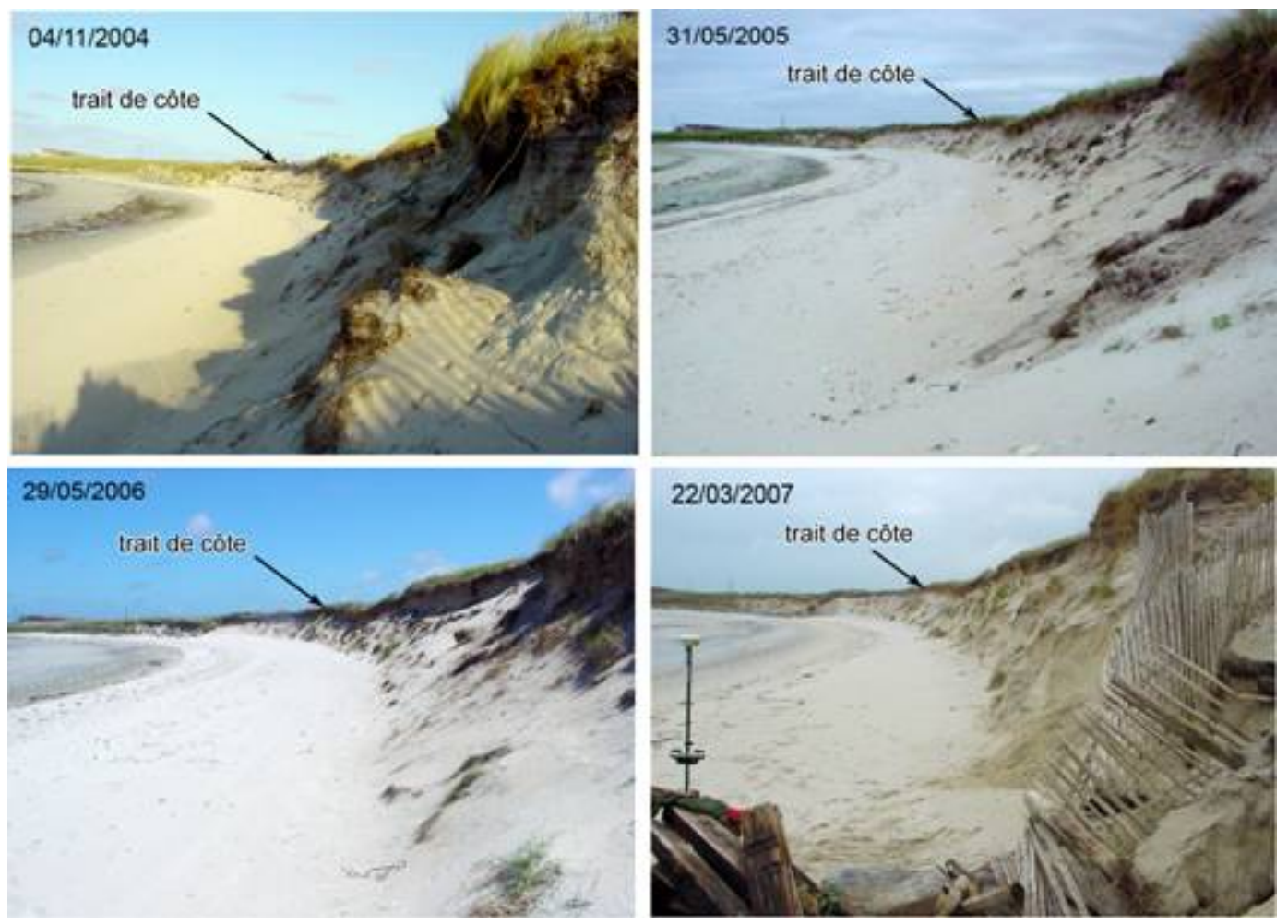

Figure 2. Profil de falaise d'érosion de la partie orientale de la dune du Vougot.

\section{Dynamique morphosédimentaire du cordon dunaire}

Les 10 mars 2008, une tempête a touché les côtes atlantiques et de la Manche. Cet évènement tempétueux combiné à une pleine mer de vive-eau (coeff. 106) a été particulièrement morphogène (CARIOLET et al., 2010). Durant la pleine mer du soir, alors que les vents étaient orientés NW-SE, c'est principalement la côte nord du Finistère qui a été touchée. Sur le site de Guissény, le recul du front de dune, et par là même du trait de côte, a atteint -5 à -6 m suivant les secteurs (SUANEZ \& CARIOLET, 
2010) (figure 3). Ces phénomènes d'érosion ont été expliqués par l'action des niveaux d'eau extrêmes combinant la marée observée et les phénomènes de runup. Ils ont été évalués à $10 \mathrm{~m}$ NGF, soit 4 à $5 \mathrm{~m}$ supérieur à l'altitude du pied de dune (SUANEZ et al., 2012). Cette estimation est conforme à la réalité de terrain lorsque l'on sait que le sommet du cordon dunaire culminant à 11 m NGF d'altitude était par endroit submergé par le déferlement des vagues.

Postérieurement à la tempête du 10 mars 2008, le suivi morphosédimentaire a indiqué une nette tendance à la régénération dunaire comme le montrent les mesures de profils de dune réalisées le long de trois radiales (figure 4). Les volumes sédimentaires gagnés par la dune depuis la tempête ont atteint environ $+12 \mathrm{~m}^{3} / \mathrm{ml}$ le long des radiales 1 et 2 , et $+8 \mathrm{~m}^{3} / \mathrm{ml}$ pour la radiale 3 . Cette régénération dunaire commencée depuis trois ans s'est traduite par une récupération des volumes perdus lors de la tempête (entre -8,5 et $-9 \mathrm{~m}^{3} / \mathrm{ml}$ ), notamment au niveau des profils 1 et 2 .

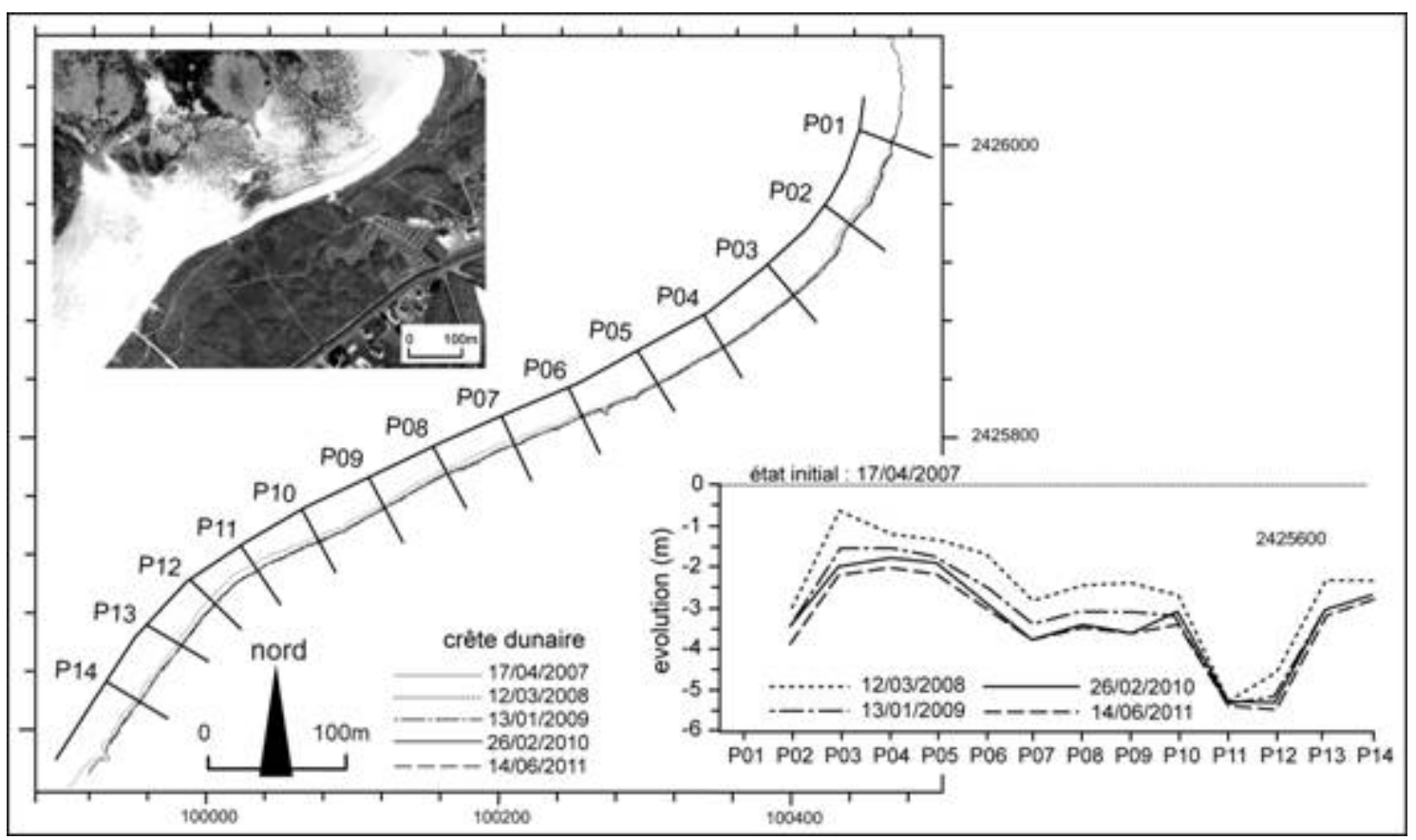

Figure 3. Cinématique du trait de côte de la plage du Vougot obtenue à partir de la limite de la crête dunaire entre le 17/04/2008 et le 14/06/2011.

Cette évolution est également confirmée par les mesures surfaciques qui ont permis d'établir un bilan sédimentaire en 3D à l'échelle de la cellule (figure 5). Entre 2009 et 2010, la dune a gagné $+1800 \mathrm{~m}^{3}$ de sable ; à cela s'ajoute près de $+12000 \mathrm{~m}^{3}$ en haut de plage intertidale, dont une partie a participé à la construction de petites dunes embryonnaires de seconde ligne (SUANEZ et al., 2012). Cette alimentation s'est faite au détriment de la section médiane de la plage intertidale qui a perdu quant à elle près de $-24000 \mathrm{~m}^{3}$ (figure $5 \mathrm{~B}$ ). Les mesures récentes réalisées dans le cadre de cette étude 


\section{XII ${ }^{\text {èmes }}$ Journées Nationales Génie Côtier - Génie Civil \\ Cherbourg, 12-14 juin 2012}

montrent que ce processus d'engraissement s'est poursuivi entre 2010 et 2011. Durant cette période la section dunaire a encore gagné environ $+7200 \mathrm{~m}^{3}$ au détriment cette fois du haut de plage intertidale qui a lui perdu $-8300 \mathrm{~m}^{3}$ (figure 5D).

Toutefois, cette tendance à la régénération morphosédimentaire du cordon dunaire ne s'est pas traduite par une progradation du trait de côte. En effet, si l'on s'en tient à la mesure du front de dune conformément au protocole suivi depuis l'année 2004, on peut voir qu'entre 2008 et 2011 la ligne de rivage a continué de reculer à des vitesses comprises entre $-0,50$ et $-0,75 \mathrm{~m} / \mathrm{an}$ (figure 3 ). Cette évolution va à l'encontre des dynamiques morphosédimentaires observées précédemment et pose dans ce cas la question de la pertinence du référentiel retenu pour la mesure du trait de côte.
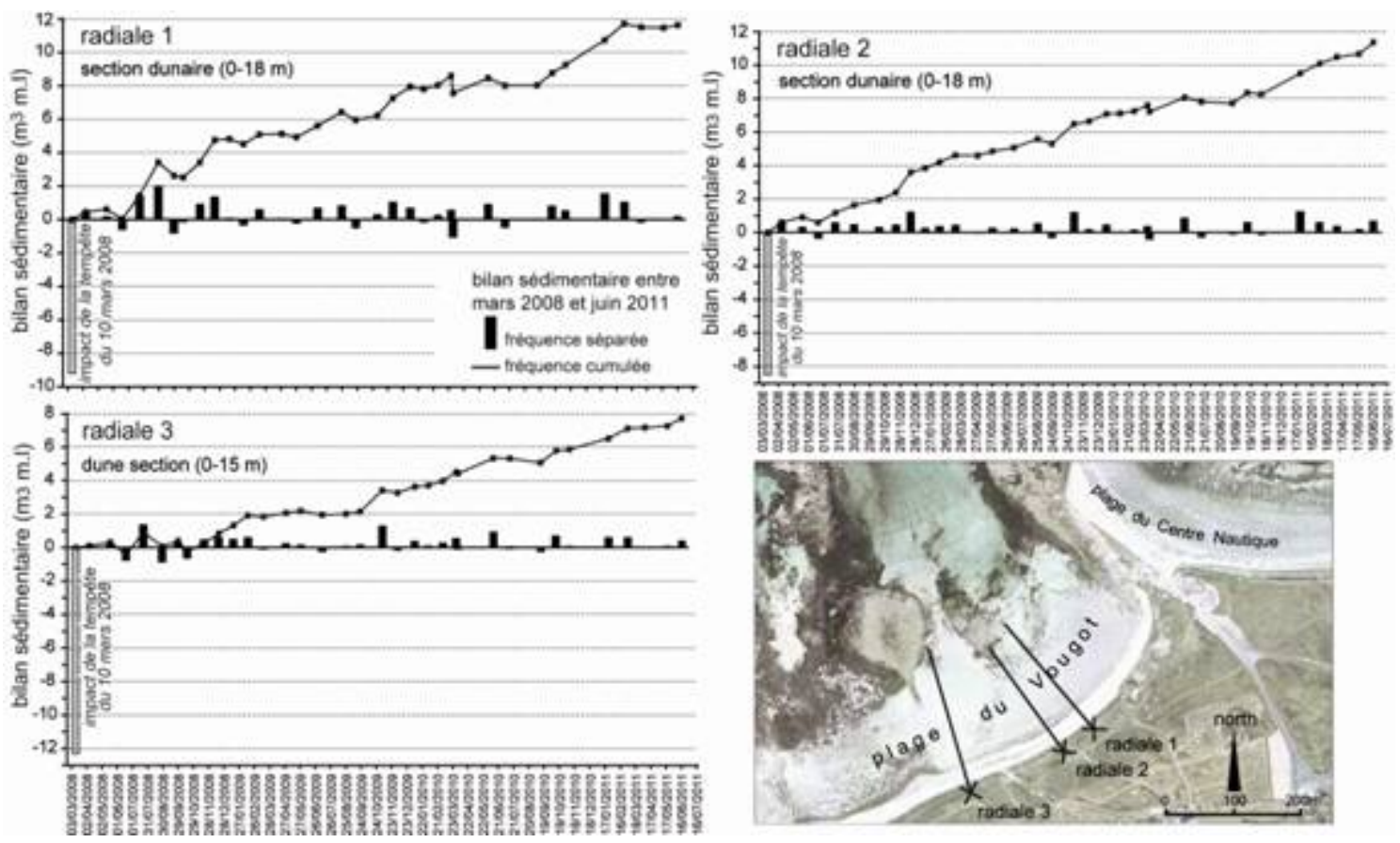

Figure 4. Evolution du bilan sédimentaire de la section dunaire (profil de dune/pied de dune) de la plage orientale du Vougot entre le 12 mars 2008 et le 16 juin 2011.

\section{Discussion et conclusion}

Le choix d'une ligne de référence du trait de côte en relation avec la dynamique morphosédimentaire et végétale des dunes n'est pas aisé. Dans le cas des cordons dunaires élevés comme pour la plage du Vougot, la régénération post-tempête des dunes ne s'accompagnent pas nécessairement d'une disparition de la falaise d'érosion et/ou d'une progradation du front de dune. A l'accumulation sableuse limitée en pied de dune succède généralement un abrupt encore bien net (figure 6). Dans ce cas, le choix de la crête dunaire (ou sommet de cet abrupt) comme ligne de référence, peut occulter les phénomènes d'engraissement sédimentaire, et par là même, de progradation du trait de côte qui s'observent en période de régénération du système littoral. 


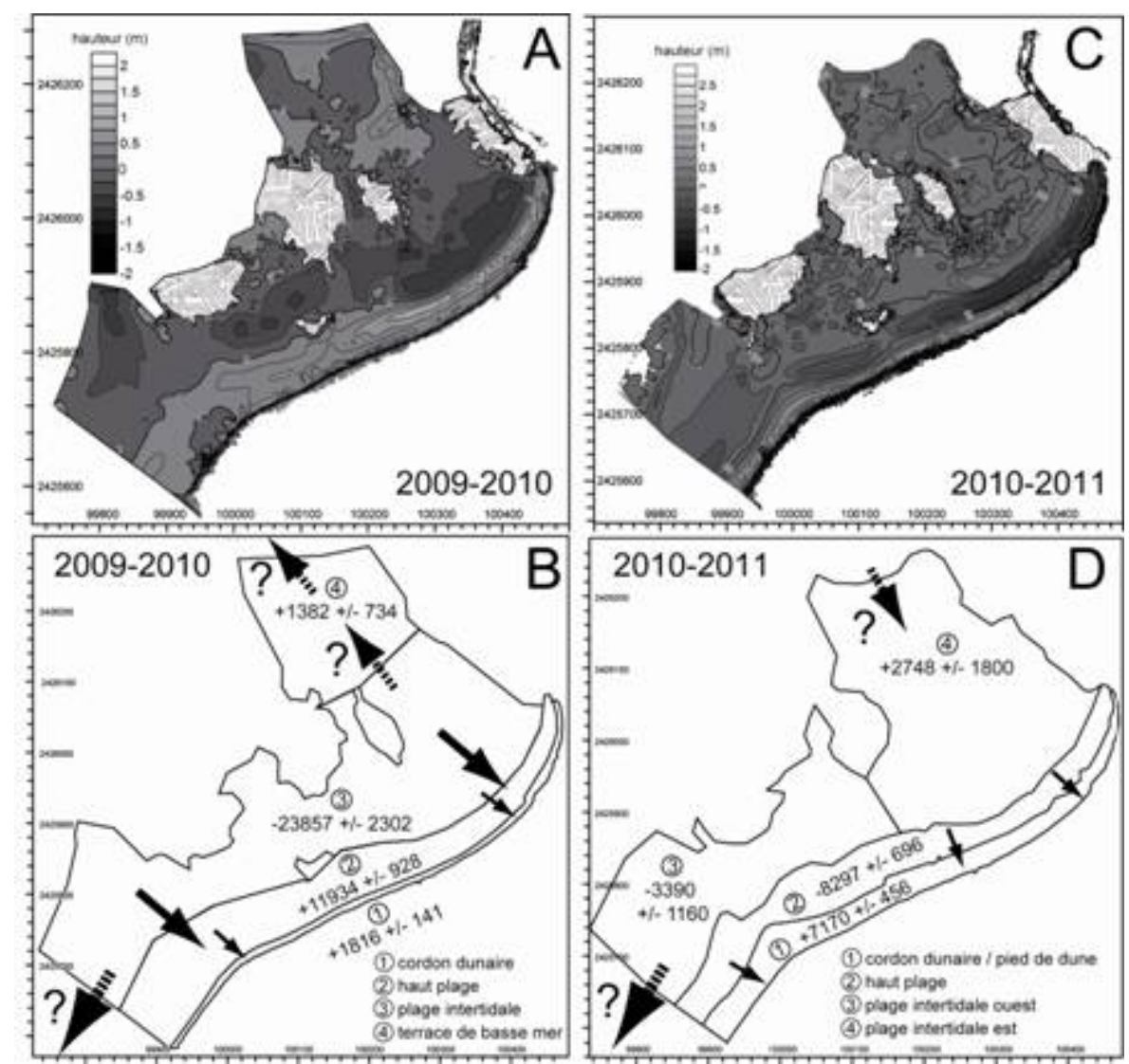

Figure 5. Evolution du bilan sédimentaire de la cellule littorale (dune/plage intertidale)

la plage orientale du Vougot entre les mois d'octobre 2009 et de juin 2011.

La limite de végétation dunaire semble alors beaucoup mieux appropriée car elle illustre plus clairement ces processus de régénération et d'avancée de la ligne de rivage (figure 6). Ceci se justifie d'autant plus lorsque l'on travaille sur des photographies aériennes qui n'offrent qu'une vision en plan. Cette limite pose toutefois la question des associations végétales que l'on retiendra entre la végétation de la dune grise, de la dune bordière, ou de la ceinture halonitrophile de pied de dune/haut de plage. Ce choix est indissociable de la dynamique végétale des dunes littorales qui est elle-même liée aux processus morphosédimentaires (HALLÉGOUËT, 1981). Dès le printemps la construction des dunes embryonnaires en pied de dune bordière est favorisée par la pousse d'espèces végétales annuelles halonitrophiles qui s'installent sur des sols riches en nitrates provenant de la décomposition des laisses de mer. Sur le littoral de la Manche et de l'Atlantique ces plantes sont principalement composées du Cakilier maritime (Cakile maritima), de l'Arroche des sables (Atriplex laciniata), et de la soude Salsola kali, (Salsola soda ou Suaeda maritima) (GÉHU, 1969). Ces plantes annuelles ont un cycle végétatif court qui s’étend du début du printemps à la fin de l'automne. Toutefois, si la construction des dunes embryonnaires s'accroît sous l'effet d'apports 


\section{XII ${ }^{\text {èmes }}$ Journées Nationales Génie Côtier - Génie Civil \\ Cherbourg, 12-14 juin 2012}

sableux, ces édifices peuvent se maintenir après la disparition des plantes halonitrophiles durant l'hiver. De nouvelles espèces végétales comme le chiendent des sables (Elymus farctus) colonisent alors la forme. Si la construction dunaire s'épaissit et s'élève jusqu'à atteindre un niveau supérieur aux pleines mers de vive-eau, l'oyat (Ammophila arenaria) s'installe. A ce stade, la ceinture de dunes embryonnaires devient pérenne et prend l'allure d'une banquette sableuse végétalisée pouvant être assimilée à une dune bordière de seconde ligne. Si l'on s'en tient à ces éléments, il convient avant tout de faire coïncider la période de mesures de terrain in situ avec celles des prises de photographies aériennes qui généralement se font durant l'été. Ainsi, la limite de végétation que l'on retiendra correspond à la ceinture halonitrophile qui est généralement bien visible sur le terrain comme sur les photos aériennes. Cela permet d'obtenir de longues séries homogènes de la cinématique du trait de côte. Si les mesures sont réalisées en hiver, on prendra la limite des chiendents ou des oyats qui reste toutefois difficile à identifier lorsque la densité du couvert végétale est faible.

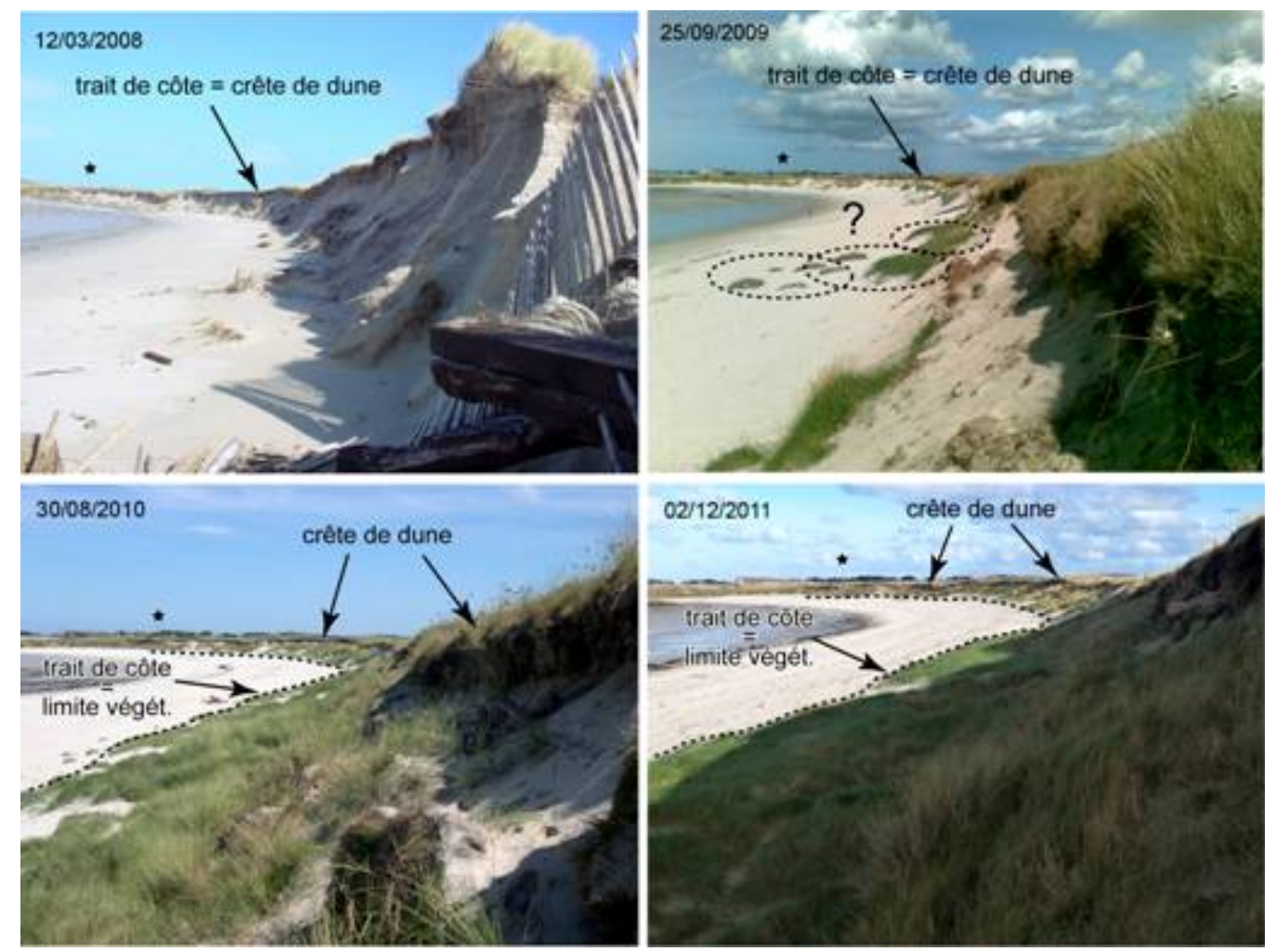

Figure 6. Régénération du cordon dunaire du Vougot et progradation du trait de côte post-tempête du 10 mars 2008.

La figure 7 montre que le choix de la ceinture halonitrophile comme "nouvelle" limite du trait de côte est bien plus approprié pour illustrer les processus de régénération du 
système dunaire. Dans ce cas, la progradation du trait de côte depuis la tempête du 10 mars 2008 a atteint $+8 \mathrm{~m}$ suivant les secteurs (cf. P03, figure 7).

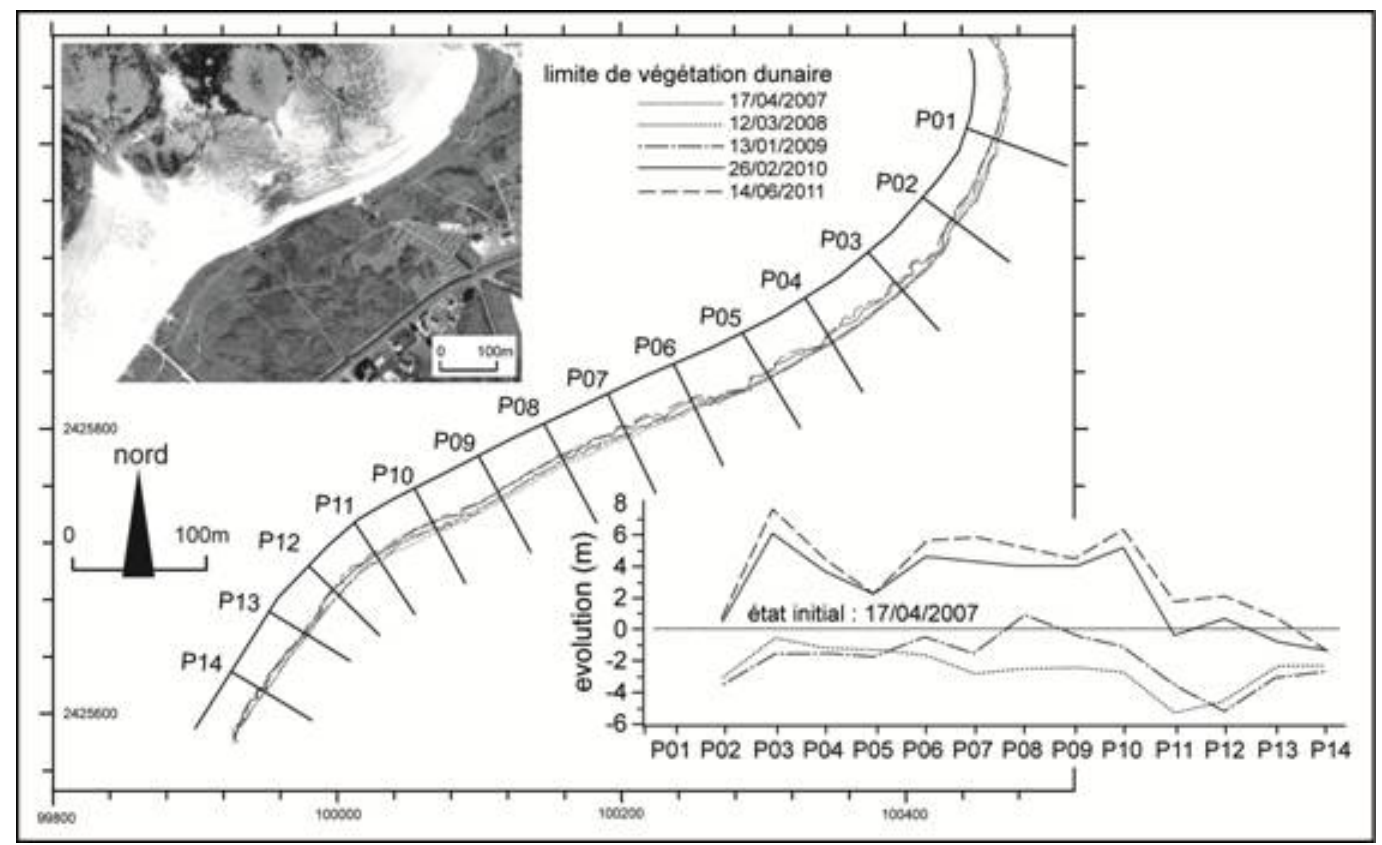

Figure 7. Cinématique du trait de côte de la plage du Vougot obtenue à partir de la limite de la ceinture halonitrophile entre le 17/04/2008 et le 14/06/2011.

\section{Références bibliographiques}

ALLAIN S., GUILLAUMONT B., LEVISAGE C., LOUBERSAC L., POPULUS J. (2000). Données géographiques de référence en domaine littoral. In POPULUS J., LOUBERSAC L. (éd.): CoastGIS'99: Geomatics and coastal environment, Shom/Ifremer, pp. 67-79.

BOAK E.H., TURNER I.L. (2005). Shoreline definition and detection: a review. Journal of Coastal Research, vol. 21, n 4, pp. 688-703. doi:10.2112/03-0071.1

CARIOLET J.-M., COSTA S., CASPAR R., ARDHUIN F., MAGNE R., GOASGUEN G. (2010). Aspects météo-marins de la tempête du 10 mars 2008 en Atlantique et en Manche. Norois, $\mathrm{n}^{\circ} 215$, pp. 11-31.

GAILLOT S., CHAVEROT S. (2001). Méthode d'étude des littoraux à faible évolution. Cas du delta du Golo (Corse) et du littoral du Touquet (Pas de calais) en France. Géomorphologie : relief, processus, environnement, $\mathrm{n}{ }^{\circ} 1$, pp. 47-54.

GÉHU J.-M. (1969). Essai synthétique sur la végétation des dunes armoricaines, Penn ar Bed, ${ }^{\circ} 57$, pp. 25-48.

GRENIER, A., DUBOIS, J.M.M. (1990). Evolution littorale récente par télédétection : synthèse méthodologique. Photo-Interprétation, vol. 6, pp. 3-7.

GUILCHER A., HALLÉGOUËT B. 1991. Coastal dunes in Brittany and their management. Journal of Coastal Research, vol. 7, n 2, pp. 517-533. 


\section{XII ${ }^{\text {èmes }}$ Journées Nationales Génie Côtier - Génie Civil \\ Cherbourg, 12-14 juin 2012}

HALLÉGOUËT B. (1981). Les crêtes dunifiées du Massif armoricain : formation et évolution. Géographie Physique Quaternaire, n 35, pp. 205-218.

LEATHERMAN S.P. (2003). Shoreline change mapping and management along U.S. East Coast. Journal of Coastal Research, vol. 38 (SI), pp. 5-13.

LE BERRE I., HÉNAFF A., DEVOGÈLE T., MASCRET A., WENZEL F. (2005). SPOT5 : un outil pertinent pour le suivi du trait de côte ? Norois, $\mathrm{n}^{\circ}$ 196, pp. 23-35.

ROBIN M. (2002). Télédétection et modélisation du trait de côte et de sa cinématique. In BARON-YELLES N., GOELDNER-GIANELLA L., VELUT S. (éd.) : Le littoral, regards, pratiques et savoirs. Editions Rue d'Ulm, Presses universitaires de l'Ecole Normale Supérieure, Paris, pp. 95-115.

SUANEZ S., STÉPHAN P. (2006) - Forçages météo-marins et dynamique morphosédimentaire saisonnière des cordons dunaires. Exemple de la baie de SaintMichel-en-Grève (Côtes d'Armor, Bretagne), Géomorphologie : relief, processus, environnement, $\mathrm{n}^{\circ} 2$, pp. 91-110.

SUANEZ S., CARIOLET J.-M. (2010). L'action des tempêtes sur l'érosion des dunes : les enseignements de la tempête du 10 mars 2008, Norois, ${ }^{\circ}$ 215, pp. 77-99.

SUANEZ S., CARIOLET J.-M., FICHAUT B. (2010). Monitoring of Recent Morphological Changes of the Dune of Vougot Beach (Brittany, France) Using Differential GPS, Shore \& Beach, vol. 78, n 1, pp. 37-47.

SUANEZ S., STÉPHAN P. (2011). Effects of Natural and Human Forcing on Mesoscale Shoreline Dynamics of Saint-Michel-en-Grève Bay (Brittany, France). Shore \& Beach, vol. 79, n² 2, pp. 19-38.

SUANEZ S., CARIOLET J.-M., CANCOUËT R., ARDHUIN F., DELACOURT C. (2012). Dune recovery after storm erosion on a high-energy beach: Vougot beach, Brittany (France), Geomorphology, vol. 139-140, pp. 16-33. doi:10.1016/j.geomorph.2011.10.014 
Thème 2 - Dynamique sédimentaire 